\title{
Structure and magnetic properties of sputtered thin films of $\mathrm{Fe}_{0.79} \mathrm{Ge}_{0.21}$
}

H. H. Hamdeh

Department of Physics, Wichita State University, Wichita, Kansas 67260

S. A. Oliver

Center for Electromagnetics Research, Northeastern University, Boston, Massachusetts 02115

B. Fultz and Z. Q. Gao

Mail 138-78, California Institute of Technology, Pasadena, California 91125

(Received 28 May, 1993; accepted for publication 2 July 1993)

Films of $\mathrm{Fe}_{0.79} \mathrm{Ge}_{0.21}$ with thicknesses of $300 \mathrm{~nm}$ were synthesized by ion beam sputtering, and were annealed at temperatures from 200 to $550^{\circ} \mathrm{C}$. The materials were characterized by $\mathrm{x}$-ray diffractometry, Mössbauer spectrometry, vibrating sample magnetometry, ferromagnetic resonance spectrometry, and electrical resistivity measurements. The as-prepared materials comprised chemically disordered bcc crystallites of sizes less than $20 \mathrm{~nm}$, and were found to have a distribution of internal strains. Upon annealing at temperatures of $250^{\circ} \mathrm{C}$ and below, there occurred strain relaxation, some evolution of short range chemical order, and an improvement in soft magnetic properties. The coercive field was a minimum for the sample annealed at $250^{\circ} \mathrm{C}$. Crystallite growth occurred at higher annealing temperatures, accompanied by a transition in several measured parameters from those of ultrafine grained materials to those typical of polycrystalline materials. This trend can be explained with the random anisotropy model. Mössbauer and magnetization measurements indicated that the Ge atoms behave as magnetic holes. The ${ }^{57} \mathrm{Fe}$ hyperfine magnetic field distribution, and its change during chemical ordering, can be calculated approximately with a model of magnetic response. The large local isomer shifts at ${ }^{57} \mathrm{Fe}$ atoms near $\mathrm{Ge}$ atoms suggest that a local depletion of $4 s$ conduction electron density should be incorporated into the model.

\section{INTRODUCTION}

In the field of magnetic materials, a subtopic of recent interest is the magnetic properties of materials with nanoscale microstructures. The magnetic properties of nanophase materials can be tuned from soft to hard, depending on the magnetic coupling between their small crystallites. In particular, there is widespread interest in an extremely soft magnetic material, "Finemet," which is produced commercially by the dense precipitation of nanocrystalline bcc $\mathrm{Fe}-\mathrm{Si}$ within an amorphous matrix. ${ }^{1,2}$ Soft magnetic properties of large-grained polycrystals were studied previously for alloys of $\mathrm{Fe}-\mathrm{Ge}$ and $\mathrm{Fe}-(\mathrm{Al}, \mathrm{Si})-\mathrm{Ge}^{3-5}$ and with metalloid substitutions the magnetic properties were studied in the amorphous state., ${ }^{6,7}$ Noteworthy is the slightly negative magnetostriction of $\mathrm{Fe}-\mathrm{Ge}$ alloys with compositions of $20-25$ at. $\% \mathrm{Ge}^{4,5}$

Here we report results of a study on the microstructure and the magnetic properties of nanocrystalline $\mathrm{Fe}-\mathrm{Ge}$ alloys; specifically the change in magnetic properties upon annealing, and their correlation to changes in the structure of the materials. Our thin films of $\mathrm{Fe}-\mathrm{Ge}$ were made by sputtering. Their composition of 79.5 at. \% $\mathrm{Fe}-21.5$ at. \% Ge lies near the low-temperature phase field of the bcc-based $D_{3}$ ordered structure, ${ }^{8}$ and short- or long-range $\mathrm{DO}_{3}$ order (SRO or LRO) appeared after the as-prepared alloys were annealed at low temperatures. The strain relaxation, growth of crystallites, and the growth of chemical order were measured by X-ray diffractometry and Mössbauer spectrometry. The latter technique also provided information on local magnetism. Macroscopic magnetic pa- rameters were obtained from vibrating sample magnetometry, and ferromagnetic resonance was used for obtaining magnetic parameters and for testing the sample homogeneity. Electrical resistivity was also measured.

We found that samples annealed at higher temperatures first underwent a relaxation of strains and the growth of chemical SRO. For annealings at progressively higher temperatures but below $300^{\circ} \mathrm{C}$, there was a corresponding decrease in coercive field and an improvement in the quality of ferromagnetic resonance (FMR) spectra. In addition, both magnetization and Mössbauer spectrometry measurements indicated that the easy axis of film magnetization tended away from its initially normal orientation, and toward the plane of the film. For samples annealed at temperatures above $300^{\circ} \mathrm{C}$, however, there was an increase in coercive field and a degradation in the ferromagnetic resonance spectra toward values typical of polycrystalline materials. A change was also observed in the orientation of magnetization, as the earlier trend was reversed and the sample plane became harder magnetically due to textured crystallite growth. This transition coincided with the ongoing increase in average crystallite size, suggesting that the soft magnetic properties after the $250^{\circ} \mathrm{C}$ annealing may be partially understood with the model of random anisotropy ${ }^{9}$ that has been used to interpret the soft magnetic properties of Finemet. ${ }^{2}$

The ${ }^{57} \mathrm{Fe}$ hyperfine magnetic field distribution was obtained from Mössbauer spectra by assuming a strong correlation between local hyperfine magnetic field and local isomer shift. The large local isomer shifts suggest that a 
local depletion in $4 s$ conduction electron density should be considered when calculating the hyperfine magnetic fields within the magnetic response model. ${ }^{10-12}$

\section{EXPERIMENT}

\section{A. Sample preparation}

The Fe-Ge thin films were deposited onto a $5.1 \mathrm{~cm}$ square optically polished fused quartz substrate by standard ion beam sputtering. A mosaic sputtering target was used, consisting of a $10 \mathrm{~cm}$ diam polycrystalline 99.999 at. \% Ge plate overlapped by a pair of 99.998 at. \% Fe foils. The area of the surface impacted by the beam was approximately $25 \% \mathrm{Ge}$ and $75 \% \mathrm{Fe}$. The substrate was positioned slightly off axis, and rotated at $3 \mathrm{rpm}$ in order to minimize film columnar growth and self-shadowing effects. The system base pressure before sputtering was $8 \times 10^{-8}$ Torr while the pressure of gettered argon gas during sputtering was $1.1 \times 10^{-4}$ Torr. The maximum temperature of the film during deposition was $50^{\circ} \mathrm{C}$. A film thickness of $280 \pm 8 \mathrm{~nm}$ was measured by thickness profilometer measurements on a step-masked portion of the film. The film composition was measured by a Kevex energy dispersive $x$-ray spectrometer mounted on a JEOL 6800 scanning electron microscope, and by an EDAX 9900 energy dispersive $x$-ray spectrometer mounted on a Philips EM430 transmission electron microscope. Samples were cored from the deposited film, and $1 \mathrm{~cm}$ diam samples were used for Mössbauer spectrometry, x-ray diffractometry, and electrical resistivity measurements, and $0.5 \mathrm{~cm}$ diam samples were used for vibrating sample magnetometry and ferromagnetic resonance spectrometry measurements. Samples were annealed in a flowing-gas tube furnace using gettered ultrapure argon gas and a quartz lamp heat source. The typical annealing procedure was as follows: the sample was allowed to warm in the convective flow away from the center for 5-10 min to avoid thermal shock, then placed in the center of the furnace for a 15 min soak period. Upon removal from the center of the furnace, the sample was placed in the original position for a $10 \mathrm{~min}$ period to cool in the flowing gas.

\section{B. Measurements}

$\mathrm{X}$-ray diffractometry was performed with an Inel CPS120 diffractometer system using monochromatized Co $K \alpha$ radiation and a curved position sensitive detector (PSD) spanning $120^{\circ}$ in $2 \theta$ angle. For this diffractometer system the angle of incidence was fixed (typically at $14^{\circ}$ ), so the direction of $\Delta \mathbf{k}$ was different for each diffraction peak. Mössbauer spectra were obtained at room temperature using a conventional constant acceleration spectrometer. A radiation source of $30 \mathrm{mCi}{ }^{57} \mathrm{Co}$ in a $\mathrm{Rh}$ matrix was used, and conversion electron spectra were obtained with a $\mathrm{He}-\mathrm{CH}_{4}$ flow-gas detector.

Magnetization measurements were performed on a Digital Measurement Systems vibrating sample magnetometer. Hysteresis loops were taken along the film normal at magnetic fields to $17.5 \mathrm{kOe}$, providing values for sample saturation magnetization, $4 \pi M_{s}$. The effective magnetiza-

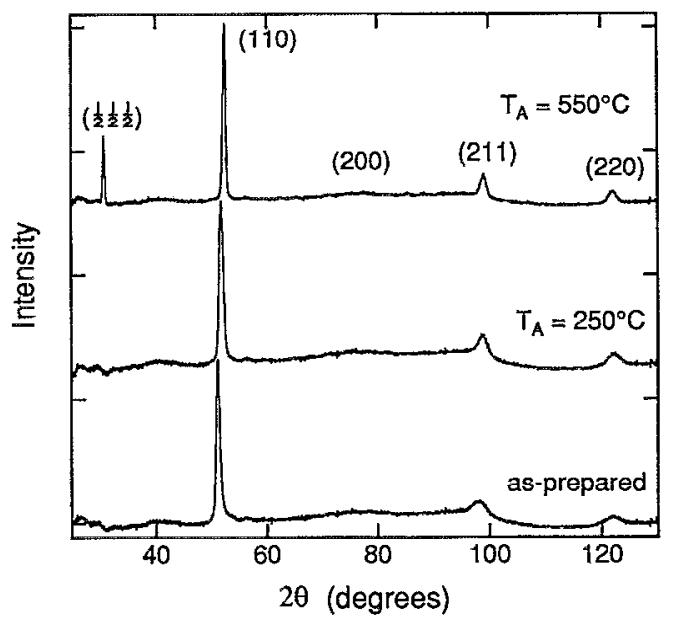

FIG. 1. X-ray diffraction pattern of the as-prepared and two annealed samples of sputtered-Fe $\mathrm{F}_{0.79} \mathrm{Ge}_{0.21}$ thin films.

tion $\left(4 \pi M_{\text {eff }}\right)$, which measures the total demagnetizing field normal to the sample plane, was evaluated from the hysteresis loop using the projected point of intersection between the linear low field magnetization behavior and the saturation magnetization. In-plane hysteresis loops provided values for $4 \pi M_{s}$, the remanent magnetization $\left(4 \pi M_{r}\right)$, the in-plane anisotropy field, and the coercive field, $H_{c}$.

Ferromagnetic resonance (FMR) measurements were performed on an X-band electron spin resonance spectrometer using a $\mathrm{TE}_{102}$ cavity at magnetic fields to $21 \mathrm{kOe}$. FMR spectra were obtained with the applied magnetic field, lying both in the sample plane and along the sample normal. Extracted parameters include values for $4 \pi M_{\text {eff }}$ and an estimate of the in-plane anisotropy field. Measurements with the applied magnetic field along the sample normal, provided the spin wave resonances (SWR) spectra of the samples. Electrical resistivities of the samples were measured with a linear four point probe method, with corrections for the finite sample size and probe spacing. ${ }^{13}$

\section{RESULTS AND DISCUSSION}

\section{A. X-ray diffractometry}

Representative $\mathrm{x}$-ray diffraction patterns are presented in Fig. 1. These data and all others showed only fundamental diffractions of the bcc lattice, and none from the hexagonal $B 8_{1}$ structure, even though some $B 8_{1}$ phase could be expected in equilibrium. ${ }^{8}$ Superlattice diffractions of the bcc-based $D 0_{3}$ ordered structure were observed from samples annealed at the higher temperatures. The $\left(\frac{1}{2} \frac{1}{2} \frac{1}{2}\right)$ superlattice diffraction peak of the $\mathrm{DO}_{3}$ ordered structure was first seen in the diffraction pattern from the sample annealed at $400^{\circ} \mathrm{C}$, and its intensity grew with annealing temperature, approximately doubling in intensity for the specimen annealed at $550^{\circ} \mathrm{C}$. [For samples annealed at $350^{\circ} \mathrm{C}$, however, the $\left(\frac{1}{2} \frac{1}{2} \frac{1}{2}\right)$ peak was observed after annealing for several hours.] The fundamental diffraction peaks became sharper with increased annealing temperature, ow- 

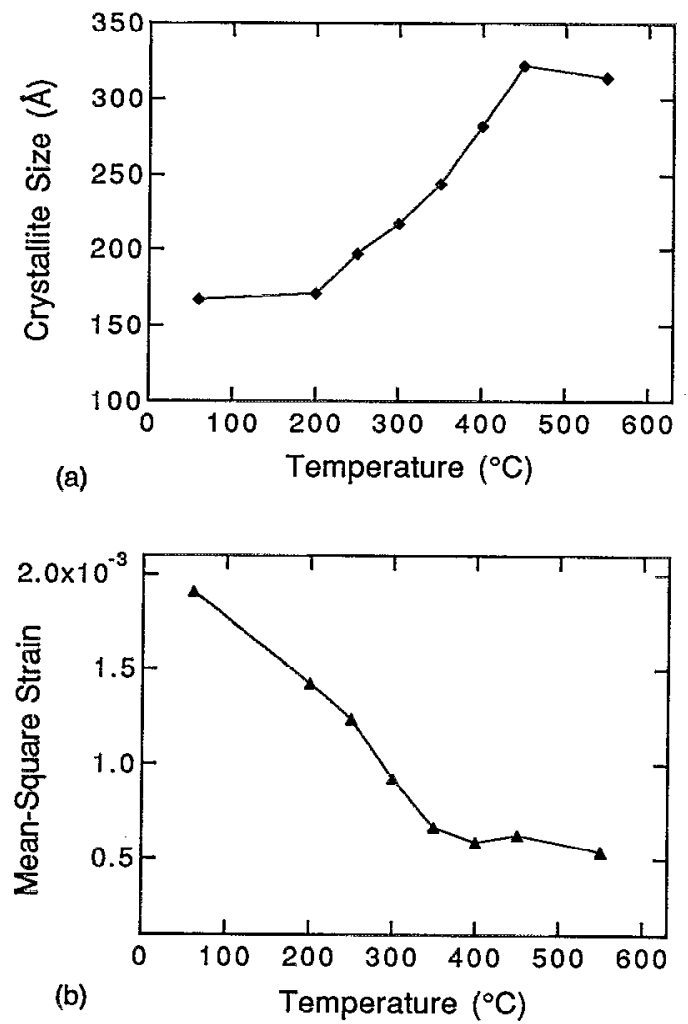

FIG. 2. (a) Crystallite size and (b) mean-square strain obtained from widths of the $x$-ray diffraction peaks vs annealing temperature.

ing to strain relaxation and grain growth. The breadths of the fundamental peaks were used to estimate the meansquared strain and the grain size by plotting the $k$-space width of a Lorentzian fit to each diffraction peak, versus the magnitude of $k$ for the diffraction. The slopes of these graphs were used to obtain a mean-squared strain, and the $y$-axis intercepts were used to obtain an average value for grain size. ${ }^{14}$ These data are presented in Figs. 2(a) and 2 (b). To determine the crystallographic texture, $x$-ray diffraction was also performed with various angles of the incident beam. At an incidence angle of $14^{\circ}$, the observed $\left(\frac{1}{2} \frac{1}{2} \frac{1}{2}\right)$ peak was strongest. Since the $\left(\frac{1}{2} \frac{1}{2} \frac{1}{2}\right)$ peak is at a $2 \theta$ diffraction angle of about $30^{\circ}$, we find that there is a crystallographic preference for the [111] direction to lie normal to the plane of the sample.

\section{B. Magnetometry, resistivity, and ferromagnetic resonance measurements}

The results of magnetometry, resistivity, and ferromagnetic resonance measurements are presented in Table I. Data are presented for the average saturation magnetization, effective magnetization, and the in-plane coercive field for the as-prepared and annealed samples. The saturation magnetization shows no significant variation for the annealed samples, having a value near $14 \mathrm{kG}$. This agrees with previous results for $\mathrm{Fe}-\mathrm{Ge}$ alloys, ${ }^{4}$ is comparable to results found for evaporated $\mathrm{Fe}-\mathrm{Si}$ and $\mathrm{Fe}-\mathrm{B}$ alloys having similar compositions, ${ }^{15}$ and matches the value of $14.0 \mathrm{kG}$ obtained for polycrystalline $\mathrm{Fe}_{3} \mathrm{Ge}^{16}$
TABLE I. Magnetic and electrical properties.

\begin{tabular}{|c|c|c|c|c|c|c|}
\hline $\begin{array}{l}T_{\text {anneal }} \\
\left({ }^{\circ} \mathrm{C}\right)\end{array}$ & $\begin{array}{c}\langle H\rangle \\
(\mathrm{kG})\end{array}$ & $\begin{array}{c}4 \pi M_{S} \\
(\mathrm{kG}) \\
( \pm 0.4)\end{array}$ & $\begin{array}{c}4 \pi M_{\text {eff }} \\
(\mathrm{VSM}) \\
(\mathrm{kG}) \\
( \pm 0.4)\end{array}$ & $\begin{array}{c}4 \pi M_{\text {eff }} \\
(\mathrm{FMR}) \\
(\mathrm{kG}) \\
( \pm 0.01)\end{array}$ & $\begin{array}{c}H_{C} \\
(\mathrm{Oe}) \\
( \pm 1)\end{array}$ & $\begin{array}{c}\mu \Omega \mathrm{cm} \\
( \pm 0.01)\end{array}$ \\
\hline$A-P$ & 267 & 13.9 & 13.9 & 14.05 & 66 & 112 \\
\hline 150 & 274 & 14.4 & 14.6 & 14.03 & 61 & 113 \\
\hline 200 & 267 & 14.4 & 14.4 & 14.64 & 45 & 110 \\
\hline 250 & 262 & 14.4 & 14.2 & 14.44 & 14 & 109 \\
\hline 300 & 258 & 13.8 & 14.5 & 14.47 & 19 & 100 \\
\hline 350 & 257 & 14.1 & 14.5 & 14.07 & 38 & 79 \\
\hline 400 & 257 & $\cdots$ & $\cdots$ & $\ldots$ & ... & 76 \\
\hline 450 & 257 & $\cdots$ & $\ldots$ & .. & .. & 75 \\
\hline
\end{tabular}

Neither magnetometry nor FMR measurements indicated the presence of in-plane uniaxial anisotropy for any sample. However, the shapes of the in-plane hysteresis loops for all samples are consistent with the existence of positive perpendicular anisotropy, which indicates that the easy axis of magnetization lies normal to the plane of the sample. Such anisotropy is commonly observed for relatively thick films of permalloy ${ }^{17}$ and cobalt-rich amorphous films ${ }^{18}$ having negative magnetostriction constants. It is caused by the magnetoelastic coupling to the strains in the samples. The magnitude of the perpendicular anisotropy field $\left(H_{u}\right)$ for thin films can be obtained from $H_{u}=4 \pi M_{s}-4 \pi M_{\text {eff }}$, as the presence of the easy axis normal to the film plane will reduce the effective magnetizing field given by $4 \pi M_{\text {eff }}$. Here the experimental uncertainties in $4 \pi M_{s}$ obscure determination of a value for the perpendicular anisotropy. However, a trend in the magnitude of $H_{u}$ can be observed in the ratio of remanent to saturation magnetization $\left(M_{r} / M_{s}\right)$, shown in Fig. 3. For the samples annealed at lower temperatures this ratio remains near 0.5 , indicative of positive perpendicular anisotropy. However, as the strain in the sample decreases [see Fig. 2(a)], the

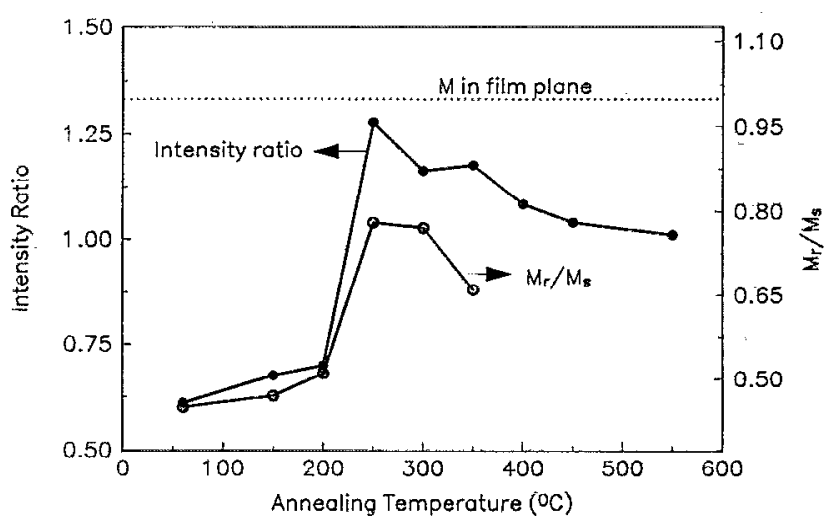

FIG. 3. Intensity ratio of the second and fifth lines of the Mössbauer spectra (left-hand scale) and ratio of the remanent magnetization to saturation magnetization (right-hand scale) of $\mathrm{Fe}_{0.79} \mathrm{Ge}_{0.21}$ thin films vs annealing temperature. 
corresponding drop in $H_{u}^{\perp}$ will make the film plane "easier," seen as an increase in the ratio $M_{r} / M_{s}$ toward the ideal easy plane value of 1 . Figure 3 indicates that $M_{r} / M_{s}$ has a maximum value occurring for the samples annealed at $250^{\circ} \mathrm{C}$. The evolution of $M_{r} / M_{s}$ for the samples annealed at still higher temperatures is driven by a different mechanism, namely the growth of textured grains having an in-plane easy axis, as discussed in Sec. III C.

The spin wave resonance spectra obtained from FMR taken normal to the plane of the sample were used as an indicator of the sample uniformity, as the mode spectra are sensitive to spatial variations in the magnetization or anisotropy field. The quality of a SWR spectrum was evaluated by the number of resolvable absorption modes, and also by the mode spacing relative to the expected spacing, which is quadratic in the mode number for standing SWR modes. The SWR spectra were found to improve for samples annealed at higher temperatures, with the best SWR spectra being obtained for the sample annealed at $300^{\circ} \mathrm{C}$. For this sample the mode behavior was approximately quadratic, and provided a deduced exchange stiffness $(A)$ of $1.5(3) \times 10^{-6} \mathrm{erg} / \mathrm{cm}$. This behavior tracks the observed decrease in strain in the samples. However, the sample annealed at $350^{\circ} \mathrm{C}$ showed only a single broad absorption mode. This mode had a very large FMR absorption linewidth $\left(\Delta H_{0}\right)$ of $160 \mathrm{Oe}$, in contrast to values of $30-50 \mathrm{Oe}$ measured for the highest field mode for all other samples. It should be noted that while the FMR linewidths of samples annealed at low temperatures are comparable to those found for amorphous iron-rich films, ${ }^{18}$ the large linewidths found for the $350^{\circ} \mathrm{C}$ samples are more typical of a polycrystalline alloy.

The trend found from FMR of improved soft magnetic properties with higher annealing temperature, followed by a degradation, was also found in the data on coercive field (see Table I). The coercive field decreases as samples were annealed at temperatures up to $350^{\circ} \mathrm{C}$, but then $H_{c}$ begins to increase for higher temperatures. The decrease in $H_{c}$ can be attributed to stress relaxation in the samples. The increase observed in $H_{c}$ and $\Delta H_{0}$, and the disruption of the SWR spectra observed for the specimen annealed at $350^{\circ} \mathrm{C}$, may be explained by the random anisotropy model ${ }^{9}$ that was used recently to explain the variation in $H_{c}$ with grain size in nanocrystalline $\mathrm{Fe}-\mathrm{Si}-\mathrm{B}-\mathrm{Cu}-\mathrm{Nb}$ alloys. ${ }^{2}$ This model predicts that the exchange interaction $(A)$ will dominate the magnetocrystalline anisotropy $\left(K_{1}\right)$ for materials having grain sizes smaller than the ferromagnetic exchange length, $L_{\mathrm{ex}}=\sqrt{\left(A / K_{1}\right)}$. This condition leads to soft magnetic behavior. Once the grain size exceeds $L_{\text {ex }}$, however, the material properties will become typical of polycrystals. For our $\mathrm{Fe}-\mathrm{Ge}$ samples the transition occurs for an average grain size of $24 \mathrm{~nm}$, from which we calculate a magnetocrystalline anisotropy of $K_{1}=3 \times 10^{6} \mathrm{erg} / \mathrm{cm}^{3}$. This value of $K_{1}$ is quite large and can be attributed to our use of an average grain size, whereas $L_{\mathrm{ex}}$ should be determined from the largest grain diameters in the sample.

Finally, the electrical resistivity data presented in Table I show a decrease that begins after annealing at $300^{\circ} \mathrm{C}$.
This decrease correlates with the onset of long range ordering and grain growth in the material.

\section{Mössbauer spectrometry-Hyperfine magnetic fields}

To obtain the hyperfine magnetic field (HMF) distribution, the experimental spectra were processed using the method of Le Caër and Dubois. ${ }^{19}$ In this method a linear relationship between the isomer shift, $I$ (with respect to pure $\mathrm{Fe}$ ), and $\mathrm{HMF}, \mathrm{H}$, is assumed:

$$
I=A H+B \text {. }
$$

The constants $A=-0.0015 \mathrm{~mm} \mathrm{~s}^{-1} \mathrm{kG}^{-1}$ and $B$ $=+0.637 \mathrm{~mm} \mathrm{~s}^{-1}$ were determined from the best fit of the calculated spectrum to the experimental one. The areas of the individual overlapping subpeaks in the HMF distributions were obtained by fitting the distribution to a set of Gaussian functions. Using an independent second method, the HMF distribution was assumed to be a sum of normalized Gaussian functions:

$$
P(H)=\sum_{i=0}^{z} \frac{p_{i}}{\sqrt{\pi} \sigma_{i}} \exp \left[-\left(\frac{H-H_{0 i}}{\sigma_{i}}\right)^{2}\right],
$$

where $p_{i}$ is a normalized probability for a specific nearneighbor environment, $\left(\Sigma_{i=0}^{z} p_{i}=1\right)$, and $\sigma_{i}$ is a measure of the width of the $i$ th Gaussian function. We used $z=8$ so that a Gaussian peak was associated with each possible number of $\mathrm{Ge}$ atoms in the $1 \mathrm{nn}$ shell of a ${ }^{57} \mathrm{Fe}$ atom. The HMF distribution was then convolved with a sextet of Lorentzian lines, with width and relative separations characteristic of a pure Fe spectrum. The isomer shift for each sextet, $I_{i}$, and the parameters $p_{i}, H_{0 i}$ and $\sigma_{i}$ were freely adjusted in the fitting routine. The values of $p_{i}$ obtained with this second method were in good agreement with our results from the method of LeCaër and Dubois. With the HMF distribution, $P(H)$, we calculated the mean HMF, $\langle H\rangle$, as

$$
\langle H\rangle=\int_{0}^{\infty} H P(H) d H,
$$

and these data are presented in Table I. During annealing, the local environments around the ${ }^{57} \mathrm{Fe}$ atoms undergo a modest change as the alloy develops chemical order. In an alloy of $\mathrm{Fe}_{3} \mathrm{Ge}$, the average number of $\mathrm{Ge}$ atom neighbors about a ${ }^{57} \mathrm{Fe}$ atom can increase from 2 to $8 / 3$ upon ordering. Upon annealing, the average HMF was reduced by about $10 \mathrm{kG}$, as expected, since the extra Ge atoms should cause a reduction in HMF, but the saturation magnetization was essentially unchanged. These small changes in $M_{S}$ and $\langle H\rangle$ upon ordering suggest that $\mathrm{Ge}$ atoms act as magnetic holes in the bcc Fe lattice, and cause little disturbance to the magnetic moments at the $\mathrm{Fe}$ atoms themselves.

The relative intensities of the second and fifth peaks of the sextets were obtained in the course of obtaining the HMF distribution. Figure 3 shows the relative intensity of the second and fifth Mössbauer peaks with respect to the first and sixth peaks as a function of annealing temperature. It is well known that this intensity ratio depends on 


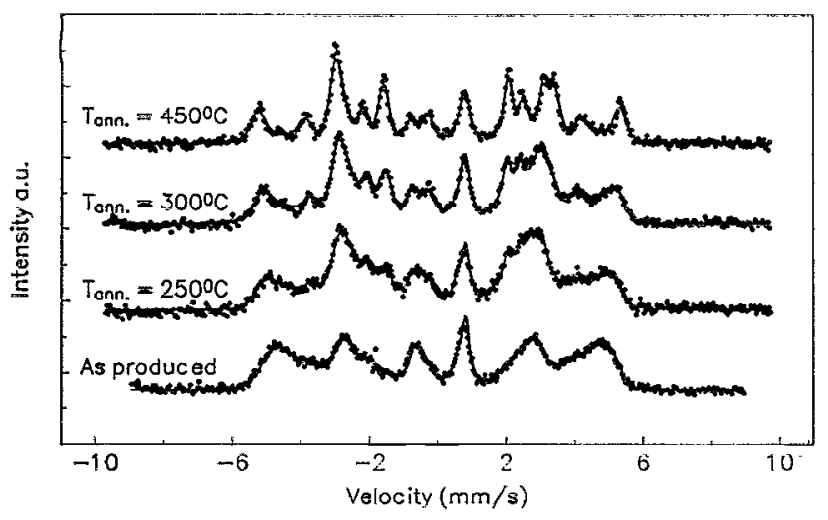

FIG. 4. Mössbauer spectra of the sputtered $\mathrm{Fe}_{0.79} \mathrm{Ge}_{0.21}$ thin films as prepared, and after annealing. The solid line was recalculated from the $P(H)$ curves of Fig. 5 .

the angle between the direction of the $\gamma$-ray beam and the direction of magnetization, and can vary between 0 (when the two directions are parallel) to $4 / 3$ (when the two directions are perpendicular). For our experimental geometry, where the $\gamma$-ray beam is normal to the plane of the thin film, the Mössbauer results suggest that the directions of magnetization were oriented randomly in the as-prepared sample. After annealing, the average magnetization rotated toward the plane of the film, achieving a maximum inplane component for the sample annealed at $250^{\circ} \mathrm{C}$. A large in-plane component is consistent with the reduction in external field energy that is possible with a soft magnetic material. At higher annealing temperatures the average magnetization tended to rotate back out of the plane of the film. Grain growth at these higher annealing temperatures would tend to emphasize the magnetic anisotropy of the individual crystallites. An easy axis of magnetization along the [100] direction (which is the easy axis of magnetization of both bec $\alpha-\mathrm{Fe}$ and the cubic phase of $\left.\mathrm{Fe}_{3} \mathrm{Ge}\right)^{20}$ should force some component of the magnetization out of the plane of the film if the films were isotropic, or if they had some [111] crystallographic texture, as determined by $\mathrm{x}$-ray diffractometry.

Typical Mössbauer spectra are shown in Fig. 4, with their HMF distributions in Fig. 5. Distinct peaks in the HMF distribution correspond to the different numbers of first nearest neighbor (1nn) Ge atoms about ${ }^{57} \mathrm{Fe}$ atoms. Previous work with bcc $\mathrm{Fe}-\mathrm{Ge}$ alloys ${ }^{21,22}$ showed that each Ge atom in the $1 \mathrm{nn}$ or $2 \mathrm{nn}$ shell of a ${ }^{57} \mathrm{Fe}$ atom perturbed the ${ }^{57} \mathrm{Fe}$ HMF by $\Delta H_{1}=+23.2 \mathrm{kG}$ and $\Delta H_{2}=+8.1 \mathrm{kG}$. The large perturbation from the $1 \mathrm{nn}$ Ge atoms can be used to calculate approximately the positions of the peaks of the HMF distribution, assuming that Ge atoms in the $2 \mathrm{nn}$ and 3nn shells of $\mathrm{Fe}$ are expected to slightly modify the HMF peak positions and widths. Further knowledge from $\mathrm{x}$-ray diffractometry that there is strong $\mathrm{DO}_{3}$ order after annealing at temperatures above $400^{\circ} \mathrm{C}$ allows us to identify the strongly growing peaks in the HMF distribution with the two ${ }^{57} \mathrm{Fe}$ sites in the $\mathrm{DO}_{3}$ structure, one of population $1 / 3$ (minority site) having (0) $1 \mathrm{nn} \mathrm{Ge}$ atoms, and the second with population $2 / 3$ (majority site) having (4) $1 \mathrm{nn} \mathrm{Ge}$

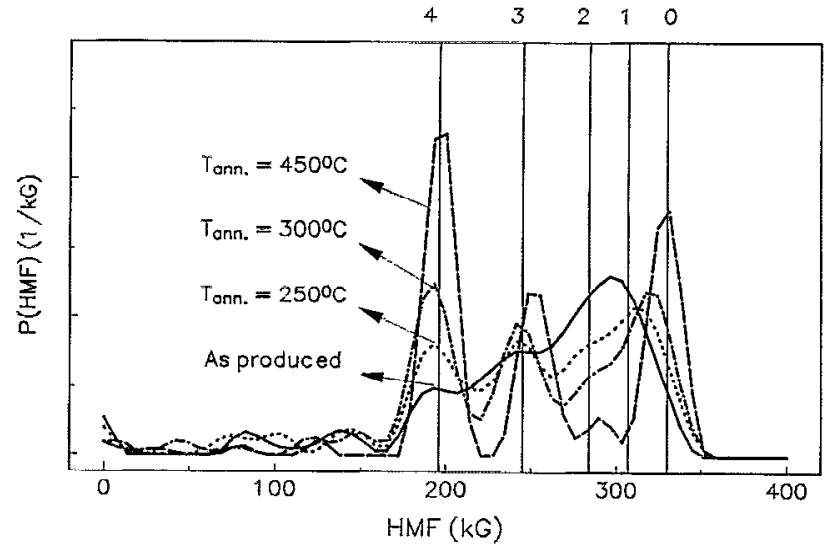

FIG. 5. HMF distributions obtained from the Mössbaur spectra of Fig. 4 by the method of Le Caër and Dubois. The peaks are identified by the number of $\mathrm{Ge}$ atoms in the $\mathrm{Inn}$ shell of ${ }^{57} \mathrm{Fe}$ as labeled at the top of the figure.

atoms. Using these two pieces of information from the dilute alloy Mössbauer spectra and from our x-ray diffractometry study, the HMF's associated with the different numbers of $1 \mathrm{nn}$ Ge atoms are labeled at the top of Fig. 5. Since the peaks of the two ${ }^{57} \mathrm{Fe}$ environments with $(0)$ and (1) $1 \mathrm{nn}$ Ge atoms are not resolved, we refer to their combination as the $(0,1) 1 \mathrm{nn}$ Ge atom peak.

The populations of the ${ }^{57} \mathrm{Fe}$ sites having different numbers of Ge neighbors [the $p_{i}$ in Eq. (2)] are parameters that describe the short-range order (SRO) in the alloy. The evolution of these SRO parameters with annealing temperature is presented in Fig. 6. We know that the populations of the different ${ }^{57} \mathrm{Fe}$ neighborhoods in a fully disordered alloy are binomial probabilities:

$$
p_{i}=\frac{8 !}{i !(8-i) !} c^{i}(1-c)^{8-i},
$$

where $c=0.21$ for our alloy. These values of $p_{i}$ are included on the left side of Fig. 6, where the closed circle is for a

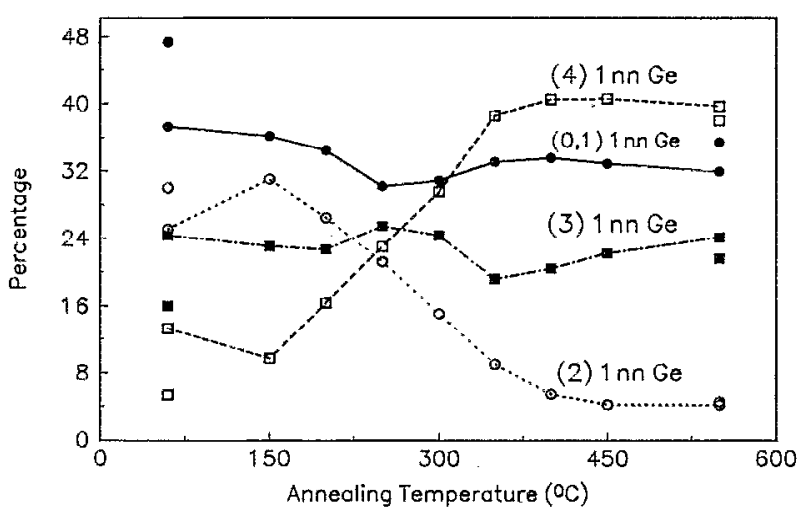

FIG. 6. Populations of the ${ }^{57} \mathrm{Fe}$ sites vs annealing temperature, determined from the areas under the peaks of the HMF distribution. The disconnected leftmost points were obtained from the binomial distribution from an alloy of $\mathrm{Fe}-21 \% \mathrm{Ge}$. The points at the right were obtained for a perfectly $\mathrm{DO}_{3}$-ordered structure of $\mathrm{Fe}-21.75 \% \mathrm{Ge}$, assuming the extra $\mathrm{Fe}$ atoms occupied randomly the Ge sites. 
TABLE II. Local isomer shifts.

\begin{tabular}{cccccc}
\hline \hline $\begin{array}{c}\text { Ge } \\
\text { neighbors }\end{array}$ & 0 & 1 & 2 & 3 & 4 \\
\hline $\mathrm{I}(\mathrm{mm} / \mathrm{s})$ & 0.142 & 0.176 & 0.210 & 0.262 & 0.343 \\
\hline
\end{tabular}

combination of the (0) and (1) $1 \mathrm{nn}$ Ge environments: $p_{1,0}=p_{0}+p_{1}$. The initial state of the alloy is partially disordered, although there is some SRO in the alloy. This SRO is characteristic of the $\mathrm{DO}_{3}$ structure; the as-prepared material has a noticeable excess of the (4) Ge environment (the majority site of the $\mathrm{DO}_{3}$-ordered structure), and probably a deficit of the (1) Ge environment (which is not expected in the $\mathrm{DO}_{3}$ ordered structure).

Although we know by inspection the populations of the two different sites in fully $\mathrm{DO}_{3}$-ordered $\mathrm{Fe}_{3} \mathrm{Ge}$, there is a substantial modification of these populations when the alloy is substoichiometric. In particular, those ${ }^{57} \mathrm{Fe}$ atoms that would have had (4) $1 \mathrm{nn} \mathrm{Ge}$ atoms now have some of those $\mathrm{Ge}$ atoms replaced with $\mathrm{Fe}$ atoms. If we assume that these extra $\mathrm{Fe}$ atoms are distributed at random over the $\mathrm{Ge}$ sites, there is a probability, $\delta c$, for finding an $\mathrm{Fe}$ atom on any Ge site:

$$
\delta c=1-4 c \text {. }
$$

These extra $\mathrm{Fe}$ atoms do not affect the environments of the ${ }^{57} \mathrm{Fe}$ atoms on the minority sites of the $D \mathrm{O}_{3}$ structure (nor of other antisited $\mathrm{Fe}$ atoms), which have (0) $1 \mathrm{nn} \mathrm{Ge}$ neighbors. For those ${ }^{57} \mathrm{Fe}$ atoms on the majority sites, however, a random substitution of $\mathrm{Fe}$ atoms into their four neighboring Ge sites gives a ratio for the number of ${ }^{57} \mathrm{Fe}$ environments with (4) and (3) Ge neighbors:

$$
\frac{p_{4}}{p_{3}}=\frac{(4 ! / 4 ! 0 !) \delta c^{0}(1-\delta c)^{4}}{(4 ! / 3 ! 1 !) \delta c^{1}(1-\delta c)^{3}}=\frac{1-\delta c}{4 \delta c} \text {. }
$$

Equation (6) is a particularly sensitive measure of $\delta c$, which can then be converted into the composition of the alloy. In our ordered alloy, the ratio $p_{4} / p_{3}=1.76$, so we obtain $\delta c=0.124$ with Eq. (6) and $c=0.219$ from Eq. (5). This is in good agreement with our composition determination by x-ray fluorescence spectrometry, and may even be more accurate. All the $p_{i}$ can be determined for an off-stoichiometric alloy by employing similar arguments, and for an alloy of $c=0.219$ these $p_{i}$ are presented as isolated points on the right-hand side of Fig. 6. The experimental data show that the SRO in the alloy is highly developed for annealings at temperatures above $400^{\circ} \mathrm{C}$, consistent with the observation of strong $\mathrm{x}$-ray superlattice diffractions.

\section{Mössbauer spectrometry-Isomer shifts}

There are different isomer shifts for ${ }^{57} \mathrm{Fe}$ atoms having different numbers of $1 \mathrm{nn}$ Ge atoms. Using our two methods for extracting the HMF distribution, we found local isometer shifts I with respect to pure bcc Fe, as listed in Table II. If these local isomer shifts arise from the change in the density of $4 s$ electrons at ${ }^{57} \mathrm{Fe}$ nuclei, $N_{4}$, the rela- tionship between $I$ and $N_{4 s}$ (Ref. 23) implies changes in $N_{4,5}$ of $-0.071,-0.088,-0.105,-0.134$, and -0.175 electrons for ${ }^{57} \mathrm{Fe}$ atoms with (0), (1), (2), (3), and (4) lnn Ge atoms, respectively. These changes are large, and show that $1 \mathrm{nn}$ Ge atoms cause a significant electronic disturbance at ${ }^{57} \mathrm{Fe}$ atoms. Because the addition of $\mathrm{Ge}$ atoms reduces $N_{4 s}$ and makes the HMF more positive, the effect of a $\operatorname{lnn} \mathrm{Ge}$ atom is to deplete the spin down $4 s$ electron density at the ${ }^{57} \mathrm{Fe}$ nucleus. This local effect may not correspond to the overall effect on the $4 s$ band, however.

These local isomer shifts are consistent with Eq. (1) which was used successfully with the same values for the constants $A$ and $B$ for both ordered and disordered alloys. Intercstingly, the constants $A$ and $B$ change by a factor of 2 during the process of $D_{3}$ ordering in $\mathrm{Fe}_{3} \mathrm{Al}^{24,25}$ On the other hand, Fig. 5 shows that the peaks in the HMF distribution increased slightly during ordering in $\mathrm{Fe}_{3} \mathrm{Ge}$, whereas they remained unchanged during ordering in $\mathrm{Fe}_{3} \mathrm{Al}^{24,25}$ These peak shifts during ordering of $\mathrm{Fe}_{3} \mathrm{Ge}$ are small, however, and they suggest only small effects on the ${ }^{57} \mathrm{Fe}$ HMF distribution from $2 \mathrm{nn}$ and $3 \mathrm{nn}$ Ge atoms. Weak effects of $2 \mathrm{nn}$ or $3 \mathrm{nn}$ Ge atoms were found for those ${ }^{57} \mathrm{Fe}$ atoms with (0) $1 \mathrm{nn}$ Ge atoms, whose $2 \mathrm{nn}$ environment changes from about $1.52 \mathrm{nn}$ Ge atoms in the disordered statc to nearly $6 \mathrm{Ge} 2 \mathrm{nn}$ atoms in the ordered state. Measurements of $2 \mathrm{nn}$ HMF perturbations of dilute $\mathrm{Fe}-\mathrm{Ge}$ alloys suggest that such a change would shift the (0) $1 \mathrm{nn}$ peak by $+35 \mathrm{kG}$ to a lower magnitude of HMF, but this is not observed. This trend could not be explained by changes in the $3 \mathrm{nn}$ shell- $3 \mathrm{nn}$ Ge atoms tend to cause a positive HMF perturbation ${ }^{21,22}$ but the number of $3 \mathrm{nn}$ Ge atoms around the minority $\mathrm{Fe}$ atoms decreases during ordering. A similarly weak effect of $2 \mathrm{nn} \mathrm{Al}$ atoms was found previously for ordering in $\mathrm{Fe}_{3} \mathrm{Al}^{25}$

The systematics of Inn HMF perturbations in dilute $\mathrm{Fe}-\mathrm{Ge}$ alloys ${ }^{21,22}$ suggest that the main peaks in the ${ }^{57} \mathrm{Fe}$ HMF distribution should be separated by regular $23.2 \mathrm{kG}$ intervals, as is common for many solutes in bcc Fe. ${ }^{26}$ Figure 5 shows that this is not the case. Greater numbers of $\mathrm{Ge}$ atom neighbors cause progressively larger shifts. Stearns has attributed this trend to a saturation of the conduction electron polarization with increasing numbers of Fe neighbors. ${ }^{27}$ We do not dispute this suggestion, but we can offer another possibility. The large local isomer shifts caused by $1 \mathrm{nn}$ Ge atoms imply large local changes in $4 s$ electron density and polarization. Neighboring Ge atoms may alter the sensitivity of the ${ }^{57} \mathrm{Fe}$. HMF to neighboring magnetic moments. In the notation of the model of magnetic response in Refs. 10-12, this represents an effect of $\mathrm{Ge}$ atoms on the conduction electron response parameter, $\alpha_{\text {CEP }}$. It is possible to predict a nonlinearity in the positions of peaks in the HMF distribution through the indirect nonlocal contribution to the HMF, $H_{\mathrm{INL}}$ (Refs. $11,12)$ but we do not yet know if this approach is realistic. We are now exploring this possibility and the further possibility that these nonlinear effects may be related to the weak effects of $2 \mathrm{nn}$ Ge atoms in partially ordered Fe-21 at. $\% \mathrm{Ge}$. 


\section{SUMMARY}

As-prepared sputtered films of Fe-21 at. \% Ge (Fe-22 at. \% Ge from Mössbaur spectrometry) were bcc polycrystals having a characteristic grain size of $17 \mathrm{~nm}$ and only some SRO characteristic of the $\mathrm{DO}_{3}$-ordered structure. Upon annealing at progressively higher temprature, the first changes were a relaxation of stresses in the film, and some development of SRO. There was a simultaneous decrease in the magnetic coercive field, and a greater tendency of the magnetization to lie in the plane of the film. We believe this magnetic softening with low-temperature annealing is due primarily to stress relaxation in the sputtered films, since this change (and probably not the chemical ordering) is also consistent with observed changes in spin wave resonance modes. A minimum coercive field of 14 Oe was obtained after annealing at $250^{\circ} \mathrm{C}$.

After annealing at temperatures higher than $250^{\circ} \mathrm{C}$, the material underwent a significant grain growth and a strong development of $\mathrm{DO}_{3}$ order. There was a simultaneous increase in the magnetic coercive field, a decreased tendency of the magnetization to lie in the plane of the film, and a qualitative change in the spin wave resonance spectra. This correlation between grain growth and magnetic hardening is well explained by the random anisotropy model, assuming the ferromagnetic exchange length is 20-30 nm.

The qualitative features of Mössbauer spectra of $\mathrm{Fe}-21$ at. \% Ge could be well explained by the magnetic response model, in which the Ge atoms act as magnetic holes. The effects of $2 \mathrm{nn}$ Ge atoms on the HMF were much smaller than expected, however. We observed large local isomer shifts at ${ }^{57} \mathrm{Fe}$ nuclei with $1 \mathrm{nn}$ Ge atoms, implying a strong loss of $4 s$ down electrons at ${ }^{57} \mathrm{Fe}$ nuclei.

\section{ACKNOWLEDGMENTS}

Work at Wichita State University was supported by an award from the Research Corporation. The Caltech part of this work was supported by the U.S. Dept. of Energy under Contract No. DE-FG03-86ER45270.
${ }^{1}$ Y. Yoshizawa, S. Oguma, and K. Yamauchi, J. Appl. Phys. 64, 6044 (1988).

${ }^{2}$ G. Herzer, IEEE Trans. Magn. 26, 1397 (1990).

${ }^{3}$ S. Arajs, Phys. Status Solidi 33, 683 (1969).

${ }^{4}$ K. Hayashi, M. Hayakawa, W. Ishikawa, Y. Ochiai, H. Matsuda, Y. Iwasaki, and K. Aso, J. Appl. Phys. 61, 3514 (1987).

${ }^{5}$ F. Sato, S. Ishio, and T. Miyazaki, Phys. Status Solidi A 107, 355 (1988).

${ }^{6}$ F. E. Luborsky, J. L. Walter, and E. P. Wohlfarth, J. Phys. F 10, 959 (1980).

${ }^{7}$ M. Mitera, M. Naka, T. Masumoto, N. Kazama, and K. Watanabe, Phys. Status Solidi A 49, K163 (1978).

${ }^{8}$ Binary Alloy Phase Diagrams, edited by T. B. Massalski (ASM International, Metals Park, OH, 1990), 2nd ed.

${ }^{9}$ R. Alben, J. J. Becker, and M. C. Chi, J. Appl. Phys. 49, 1653 (1978).

${ }^{10}$ M. B. Stearns, Phys. Rev. B 9, 2311 (1974).

${ }^{11}$ H. H. Hamdeh, B. Fultz, and D. H. Pearson, Phys. Rev. B 39, 11233 (1989).

${ }^{12}$ B. Fultz, Mössbauer Spectroscopy Applied to Magnetism and Materials Science, edited by G. J. Long and F. Grandjean (Plenum, New York, in press), Chap. 1.

${ }^{13}$ H. H. Wieder, Laboratory Notes on Electrical and Galvanomagnetic Measurements (Elsevier, Amsterdam, 1979), Chap. 1.

${ }^{14}$ H. P. Klug and L. E. Alexander, X-Ray Diffraction Procedures (Wiley, New York, 1974), Chap. 9.

${ }^{15}$ P. J. Grundy, J. Magn. Magn. Mater. 21, 1 (1980).

${ }^{16}$ J. W. Drijver, S. G. Sinnema, and F. Van der Woude, J. Phys. F 6, 2165 (1976).

${ }^{17}$ H. Fujiwara and Y. Sugita, IEEE Trans. Magn. MAG-4, 22 (1968).

${ }^{18}$ V. G. Harris, S. A. Oliver, W. B. Nowak, and C. Vittoria, J. Appl. Phys. 67, 5571 (1990).

${ }^{19}$ G. LeCaër and J. M. Dubois, J. Phys. E 12, 1087 (1979).

${ }^{20}$ Y. Nakamura and R. Tahara, J. Phys. Soc. Jpn. 41, 459 (1976).

${ }^{21}$ S. M. Dubiel and W. Zinn, Phys. Rev. B 28, 67 (1983).

${ }^{22}$ L. Brossard, G. A. Fatseas, and J. L. Dorman, J. Appl. Phys. 41, 1306 (1971).

${ }^{23}$ L. R. Walker, G. K. Wertheim, and V. Jaccarino, Phys. Rev. Lett. 6, 98 (1968).

${ }^{24}$ B. Fultz, Z. Q. Gao, and H. H. Hamdeh, Hyperfine Interac. 54, 521 (1990).

${ }^{25}$ B. Fultz and Z. Q. Gao, Nucl. Instrum. Methods Phys. Res. B 76, 115 (1993).

${ }^{26}$ G. K. Wertheim, V. Jaccarino, J. H. Wernick, and D. N. E. Buchanan, Phys. Rev. Lett. 12, 24 (1964).

${ }^{27}$ M. B. Stearns, Phys. Rev. B 6, 3326 (1972). 